\title{
ON THE GENERALIZED SPECTRUM FOR SECOND-ORDER ELLIPTIC SYSTEMS
}

\author{
ROBERT STEPHEN CANTRELL AND CHRIS COSNER
}

\begin{abstract}
We consider the system of homogeneous Dirichlet boundary value problems

(*) $\quad L_{1} u=\lambda\left[a_{11}(x) u+a_{12}(x) v\right], \quad L_{2} v=\mu\left[a_{12}(x) u+a_{22}(x) v\right]$

in a smooth bounded domain $\Omega \subseteq \mathbf{R}^{N}$, where $L_{1}$ and $L_{2}$ are formally selfadjoint second-order strongly uniformly elliptic operators. Using linear perturbation theory, continuation methods, and the Courant-Hilbert variational eigenvalue characterization, we give a detailed qualitative and quantitative description of the real generalized spectrum of $(*)$, i.e., the set $\left\{(\lambda, \mu) \in \mathbf{R}^{2}:(*)\right.$ has a nontrivial solution $\}$. The generalized spectrum, a term introduced by Protter in 1979, is of considerable interest in the theory of linear partial differential equations and also in bifurcation theory, as it is the set of potential bifurcation points for associated semilinear systems.
\end{abstract}

1. Introduction. Suppose that $\Omega$ is a bounded smooth domain in $\mathbf{R}^{N}, N \geq 1$, and that $L_{i}, i=1,2$, are second-order strongly uniformly elliptic operators acting on functions from $\Omega$ into C. Consider then the system

$$
L_{1} u=\lambda\left[a_{11}(x) u+a_{12}(x) v\right], \quad L_{2} v=\mu\left[a_{21}(x) u+a_{22}(x) v\right]
$$

in $\Omega$, where $u$ and $v$ are required to satisfy homogeneous Dirichlet boundary conditions. A point $(\lambda, \mu) \in \mathbf{C}^{2}$ for which (1.1) has a nontrivial solution is called a point of the generalized spectrum for (1.1). This term was introduced in 1979 by Protter [14] for a class of problems which includes (1.1). He found that "the process for obtaining lower bounds for the spectrum of a second order system is improved substantially by the introduction of [this] generalization of the spectrum."

From a different though related point of view, generalized spectra represent the potential primary bifurcation points to associated semilinear problems. Such problems provide good examples for the recently developed multiparameter bifurcation theory (see, for example, Alexander and Antman [1, 2], Fitzpatrick, Massabo, and Pejsachowicz [10, 11], and Ize, Massabo, Pejsachowicz, and Vignoli [13]). Further, such semilinear systems determine the steady-states to reaction-diffusion systems arising in the applications. In particular, the situation when $L_{1}=L_{2}=-\Delta$ and diffusion coefficients are allowed to vary independently from equation to equation occurs frequently.

For instance, Brown and Eilbeck [3] exploit the generalized spectrum to study stabiltity properties of constant solutions to the problem

$$
u_{t}(x, t)=d_{1} \Delta u(x, t)+F(u, v), \quad v_{t}(x, t)=d_{2} \Delta v(x, t)+G(u, v)
$$

Received by the editors May 1, 1986 and, in revised form, October 6, 1986. The contents of this paper were presented by the second author at the meeting on Ordinary Differential Equations, March 22-28, 1987, sponsored by the Mathematisches Forschungsinstitut Oberwolfach.

1980 Mathematics Subject Classification (1985 Revision). Primary 35P99, 35J55. 
when the diffusion coefficients $d_{1}$ and $d_{2}$ are allowed to vary independently.

Of course, it should be noted that in the above situations, it is not the full generalized spectrum of Protter [14] but rather its restriction to $\mathbf{R}^{2}$ which is of paramount interest. We shall adopt this point of view in this article. Consequently, unless otherwise specified the term generalized spectrum will now denote only the set $\Sigma=\left\{(\lambda, \mu) \in \mathbf{R}^{2}\right.$ : (1.1) has a nontrivial solution $\}$.

The geometric properties of the generalized spectrum for (1.1) were studied in detail in Cantrell [5] in the special case when $L_{1}=L_{2}, a_{i j}, i, j=1,2$, are positive constants, and $a_{11} a_{22}-a_{21} a_{12}>0$. The generalized spectrum was determined to be the collection of hyperbolae

$$
\left\{\lambda_{n}\left(a_{22} \mu-\lambda_{n}\right) /\left(\left(a_{11} a_{22}-a_{21} a_{12}\right) \mu-\lambda_{n} a_{11}\right): n=1,2,3, \ldots\right\}
$$

where $0<\lambda_{1}<\lambda_{2}<\cdots$ are assumed to be the eigenvalues of $L_{1}$ on $\Omega$ subject to zero Dirichlet boundary data. In particular, the hyperbolae associated with $\lambda_{m}$ and $\lambda_{n}, m<n$, intersect precisely when

$$
\frac{\lambda_{m}}{\lambda_{n}} \leq \frac{\sqrt{a_{11} a_{22}}-\sqrt{a_{21} a_{12}}}{\sqrt{a_{11} a_{22}}+\sqrt{a_{21} a_{12}}} .
$$

This information is used to analyze the bifurcation phenomena for the problem

$$
\begin{aligned}
L u & =\lambda f(u, v) & & \\
L v & =\mu g(u, v) & & \text { in } \Omega, \\
u=0 & =0 & & \text { on } \partial \Omega,
\end{aligned}
$$

where $f(0,0)=0=g(0,0)$ and $(\partial f / \partial u)(0,0)=a_{11},(\partial f / \partial v)(0,0)=a_{12}$, $(\partial g / \partial u)(0,0)=a_{21}$, and $(\partial g / \partial v)(0,0)=a_{22}$.

The analysis of the generalized spectrum in this special case uses an elimination procedure which depends heavily on the fact that the $a_{i j}$ 's are constant and that the elliptic operator is the same in both equations. It is highly desirable to eliminate these restrictions both from the point of view of studying geometric properties of generalized spectra and from the point of view of applications to more general semilinear problems of the form

$$
\begin{array}{rlrl}
L_{1} u & =\lambda f(x, u, v) & \\
L_{2} v & =\mu g(x, u, v) & & \\
u=0 & =v & & \text { on } \partial \Omega,
\end{array}
$$

where $f(x, 0,0)=0=g(x, 0,0)$. The results for the special case in [5] are instructive in this regard, in that they provide a good indication of what geometric structure to expect in general. This last is especially valuable since a direct calculation of the generalized spectrum is no longer possible.

In the present article, we obtain qualitative and quantitative information about the generalized spectrum for (1.1) which is comparable to that obtained for the special case in [5]. Our major techniques are the perturbation theory of linear operators, continuation methods based on the implicit function theorem, and variational characterizations of eigenvalues as in Courant and Hilbert [9]. We shall make the following assumptions: 
(i) $L_{1}$ and $L_{2}$ are of the form

$$
L_{k} u=-\sum_{i, j=1}^{N}\left(A_{i j}^{k}(x) u_{x_{i}}\right)_{x_{j}}+A^{k}(x) u,
$$

where, for $k=1,2\left(A_{i j}^{k}(x)\right)_{i, j=1}^{N}$ is symmetric and positive definite, $A^{k}(x) \geq 0$, $A_{i j}^{k} \in C^{1+\alpha}(\bar{\Omega}), i, j=1, \ldots, N, A^{k} \in C^{\alpha}(\bar{\Omega})$, where $0<\alpha<1$.

(ii) $a_{i j} \in C^{\alpha}(\bar{\Omega})$, for $i, j=1,2$, where $0<\alpha<1$.

(iii) $a_{11}(x)>0$ on $\bar{\Omega}, a_{22}(x)>0$ on $\bar{\Omega}, a_{11}(x) a_{22}(x)-a_{21}(x) a_{12}(x)>0$ on $\bar{\Omega}$.

In addition, we shall assume for the most part that

(iv) $a_{12} \equiv a_{21}$.

We should note that while assumption (i) was not explicitly made in [5], it is assumed there that the eigenvalues of $L_{1}=L_{2}$ are all real and positive.

The remainder of this paper is structured as follows. In $\S 2$, basic qualitative features of the set are developed via the perturbation theory of compact linear operators and the implicit function theorem. More explicit quantitative information is obtained in $\S 3$. Results on the multiplicity of eigenspaces corresponding to points of the generalized spectrum are presented in $\S 4$. Finally, $\S 5$ deals with the asymptotic structure of the generalized spectrum of (1.1).

2. Qualitative properties. Consider (1.1) and let $\Sigma$ denote its generalized spectrum. For $s \in[0,2 \pi]$, let $A(s)$ denote the operator

$$
A(s)=\left(\begin{array}{cc}
\cos s & 0 \\
0 & \sin s
\end{array}\right)\left(\begin{array}{cc}
L_{1}^{-1} & 0 \\
0 & L_{2}^{-1}
\end{array}\right)\left(\begin{array}{ll}
a_{11} & a_{12} \\
a_{12} & a_{22}
\end{array}\right)
$$

where (iv) has been assumed. If (i) is assumed, standard elliptic theory implies that $L_{1}^{-1}$ and $L_{2}^{-1}$ are compact linear operators from $C_{0}^{\alpha}(\bar{\Omega})$ to $C_{0}^{1+\alpha}(\bar{\Omega})$. Consequently $A(s)$ may be viewed as a compact linear operator on $\left[C_{0}^{1+\alpha}(\bar{\Omega})\right]^{2}$ under assumptions (i) and (ii). Observe that $(\lambda, \mu) \in \Sigma$ precisely when $\lambda=(1 / t(s)) \cos s$, $\mu=(1 / t(s)) \sin s$ where $t(s) \neq 0$ is an eigenvalue of $A(s)$ for some $s \in[0,2 \pi]$.

LEMMA 2.1. Under assumptions (i)-(iv), the eigenvalues of $A(s)$ are all real.

ProOF. We shall argue only in the case $\cos s \neq 0$ and $\sin s \neq 0$, as the exceptional cases follow from analogous arguments for a single equation. Let us suppose then that $\sigma \in \mathbf{C}$ is such that the null space $N(I-\sigma A(s)) \neq\{0\}$. Then there are $u$ and $v$, not both identically zero, such that

$$
\begin{aligned}
& L_{1} u=\sigma \cos s\left(a_{11} u+a_{12} v\right) \\
& L_{2} v=\sigma \sin s\left(a_{12} u+a_{22} v\right)
\end{aligned} \quad \text { in } \Omega
$$

with

$$
u=0=v \text { on } \Omega
$$

Consequently,

$$
\tilde{L}_{1} u=\sigma\left(a_{11} u+a_{12} v\right), \quad \tilde{L}_{2} v=\sigma\left(a_{12} u+a_{22} v\right)
$$

where $\tilde{L}_{1}=(1 / \cos s) L_{1}$ and $\tilde{L}_{2}=(1 / \sin s) L_{2}$ are formally selfadjoint elliptic operators. Taking complex conjugates of the equations of (2.1) we obtain

$$
\tilde{L}_{1} \bar{u}=\bar{\sigma}\left(a_{11} \bar{u}+a_{12} \bar{v}\right), \quad \tilde{L}_{2} \bar{v}=\bar{\sigma}\left(a_{12} \bar{u}+a_{22} \bar{v}\right) .
$$


Multiplying the top equation of $(2.1)$ by $\bar{u}$ and the top equation of (2.2) by $u$, integrating, and exploiting the formal selfadjointness of $\tilde{L}_{1}$ yield

$$
0=(\sigma-\bar{\sigma}) \int_{\Omega} a_{11}|u|^{2}+\int_{\Omega} a_{12}(\sigma v \bar{u}-\overline{\sigma v} u)
$$

Similarly, we may obtain

$$
0=(\sigma-\bar{\sigma}) \int_{\Omega} a_{22}|v|^{2}+\int_{\Omega} a_{12}(\sigma u \bar{v}-\overline{\sigma u} v) .
$$

Adding (2.3) and (2.4) produces

$$
\begin{aligned}
0 & =(\sigma-\bar{\sigma})\left(\int_{\Omega} a_{11}|u|^{2}+a_{12}(u \bar{v}+v \bar{u})+a_{22}|v|^{2}\right) \\
& =(\sigma-\bar{\sigma})\left(\int_{\Omega} a_{11}|u|^{2}+2 a_{12} \operatorname{Re}(u \bar{v})+a_{22}|v|^{2}\right) .
\end{aligned}
$$

Now

$$
\begin{aligned}
\int_{\Omega} a_{11}|u|^{2}+2 a_{12} \operatorname{Re}(u \bar{v})+a_{22}|v|^{2} \\
\quad \geq \int_{\Omega} a_{11}|u|^{2}-2\left|a_{12}\right||u||v|+a_{22}|v|^{2} \\
\quad>0
\end{aligned}
$$

since $a_{11} a_{22}-\left(a_{12}\right)^{2}>0$ and $(u, v) \not \equiv(0,0)$. Hence $\sigma=\bar{\sigma}$.

LEMMA 2.2. Under assumptions (i)-(iv),

$$
\operatorname{dim} N(I-\sigma A(s))=\operatorname{dim} N\left([I-\sigma A(s)]^{2}\right)
$$

for all $\sigma \in \mathbf{R}$ and $s \in[0,2 \pi]$.

REMARK. The result is, of course, just the statement of the equality of the geometric and algebraic multiplicities of the eigenspaces associated with $(\lambda, \mu) \in \Sigma$.

PROOF. With no loss of generality, assume that $\cos s \neq 0$ and $\sin s \neq 0$. Suppose that

$$
N(I-\sigma A(s))=\operatorname{span}\left\{\left(\begin{array}{c}
\varphi_{1} \\
\psi_{1}
\end{array}\right),\left(\begin{array}{c}
\varphi_{2} \\
\psi_{2}
\end{array}\right), \ldots,\left(\begin{array}{c}
\varphi_{m} \\
\psi_{m}
\end{array}\right)\right\} .
$$

Let us denote this span by $V$. Define $\tilde{A}: V \times V \rightarrow \mathbf{R}$ by

$$
\begin{aligned}
\tilde{A}\left(\sum_{i=1}^{m} c_{i}\left(\begin{array}{c}
\varphi_{i} \\
\psi_{i}
\end{array}\right), \sum_{k=1}^{m} d_{k}\left(\begin{array}{l}
\varphi_{k} \\
\psi_{k}
\end{array}\right)\right) \\
=\int_{\Omega}\left[a_{11}\left(\sum_{i=1}^{m} c_{i} \varphi_{i}\right)+a_{12}\left(\sum_{i=1}^{m} c_{i} \psi_{i}\right)\right]\left(\sum_{k=1}^{m} d_{k} \varphi_{k}\right) \\
+\int_{\Omega}\left[a_{12}\left(\sum_{i=1}^{m} c_{i} \varphi_{i}\right)+a_{22}\left(\sum_{i=1}^{m} c_{i} \psi_{i}\right)\right]\left(\sum_{k=1}^{m} d_{k} \psi_{k}\right) .
\end{aligned}
$$

Since $a_{11} a_{22}-\left(a_{12}\right)^{2}>0, \tilde{A}$ gives an inner product on $V$. Consequently, we may choose

$$
\left(\begin{array}{c}
\varphi_{1} \\
\psi_{1}
\end{array}\right),\left(\begin{array}{l}
\varphi_{2} \\
\psi_{2}
\end{array}\right), \ldots,\left(\begin{array}{l}
\varphi_{m} \\
\psi_{m}
\end{array}\right)
$$


so that

$$
\tilde{A}\left(\left(\begin{array}{c}
\varphi_{i} \\
\psi_{i}
\end{array}\right),\left(\begin{array}{c}
\varphi_{j} \\
\psi_{j}
\end{array}\right)\right)=\delta_{i j}
$$

Next, observe that $N(I-\sigma A(s))=N(L-\Lambda A)$ where

$$
L=\left(\begin{array}{cc}
L_{1} & 0 \\
0 & L_{2}
\end{array}\right), \quad \Lambda=\left(\begin{array}{cc}
\sigma \cos s & 0 \\
0 & \sigma \sin s
\end{array}\right), \quad \text { and } \quad A=\left(\begin{array}{ll}
a_{11} & a_{12} \\
a_{12} & a_{22}
\end{array}\right) \text {. }
$$

Furthermore, if $y \in R(L-\Lambda A), y=(L \Lambda-\Lambda A \Lambda)\left(\Lambda^{-1} x\right)$ for some choice of $x$, and consequently, if $[()$,$] denotes the inner product in \left[L^{2}(\Omega)\right]^{2}$,

$$
\begin{aligned}
\left(y, \Lambda^{-1}\left(\begin{array}{c}
\varphi_{i} \\
\psi_{i}
\end{array}\right)\right) & =\left((L \Lambda-\Lambda A \Lambda)\left(\Lambda^{-1} x\right), \Lambda^{-1}\left(\begin{array}{c}
\varphi_{i} \\
\psi_{i}
\end{array}\right)\right) \\
& =\left(\Lambda^{-1} x,(L \Lambda-\Lambda A \Lambda)\left(\Lambda^{-1}\left(\begin{array}{c}
\varphi_{i} \\
\psi_{i}
\end{array}\right)\right)\right) \\
& =\left(\Lambda^{-1} x,(L-\Lambda A)\left(\begin{array}{c}
\varphi_{i} \\
\psi_{i}
\end{array}\right)\right) \\
& =0
\end{aligned}
$$

since $L \Lambda-\Lambda A \Lambda$ is selfadjoint on $\left[L^{2}(\Omega)\right]^{2}$, where the domain of $L \Lambda-\Lambda A \Lambda$ is taken to be $\left[H^{2}(\Omega) \cap H_{0}^{1}(\Omega)\right]^{2}$.

Now suppose that $[I-\sigma A(s)]^{2} z=0$. Then $(I-\sigma A(s)) z=\sum_{i=1}^{m} c_{i}\left(\begin{array}{c}\varphi_{i} \\ \psi_{i}\end{array}\right)$ for some choice of constants $\left\{c_{1}, \ldots, c_{m}\right\}$. Consequently

$$
(L-\Lambda A) z=\sum_{i=1}^{m} c_{i} \Lambda A\left(\begin{array}{c}
\varphi_{i} \\
\psi_{i}
\end{array}\right)
$$

Hence

$$
\left(\sum_{i=1}^{m} c_{i} \Lambda A\left(\begin{array}{c}
\varphi_{i} \\
\psi_{i}
\end{array}\right), \Lambda^{-1}\left(\begin{array}{c}
\varphi_{j} \\
\psi_{j}
\end{array}\right)\right)=0 \text { for } j=1, \ldots, m
$$

But

$$
\begin{aligned}
&\left(\sum_{i=1}^{m} c_{i} \Lambda A\left(\begin{array}{c}
\varphi_{i} \\
\psi_{i}
\end{array}\right), \Lambda^{-1}\left(\begin{array}{c}
\varphi_{j} \\
\psi_{j}
\end{array}\right)\right)=\left(\sum_{i=1}^{m} c_{i} A\left(\begin{array}{c}
\varphi_{i} \\
\psi_{i}
\end{array}\right),\left(\begin{array}{c}
\varphi_{j} \\
\psi_{j}
\end{array}\right)\right) \\
&=\sum_{i=1}^{m} c_{i}\left(A\left(\begin{array}{c}
\varphi_{i} \\
\psi_{i}
\end{array}\right),\left(\begin{array}{c}
\varphi_{j} \\
\psi_{j}
\end{array}\right)\right)=\sum_{i=1}^{m} c_{i} \tilde{A}\left(\left(\begin{array}{c}
\varphi_{i} \\
\psi_{i}
\end{array}\right),\left(\begin{array}{c}
\varphi_{j} \\
\psi_{j}
\end{array}\right)\right)=\sum_{i=1}^{m} c_{i} \delta_{i j}=c_{j} .
\end{aligned}
$$

Hence $c_{j}=0$ for $j=1,2, \ldots, m$, and $(I-\sigma A(s)) z=0$.

Lemmas 2.1 and 2.2 enable us to establish the following theorem, which is the fundamental qualitative observation on $\Sigma$.

THEOREM 2.3. Suppose (i)-(iv) hold and that $\operatorname{dim} N\left(I-\sigma_{0} A\left(s_{0}\right)\right)=k_{0} \geq 1$ for some $\sigma_{0} \in \mathbf{R}$ and $s_{0} \in[0,2 \pi]$. Then there is $a \delta_{0}>0$ and $k_{0}$ analytic functions from $\left(s_{0}-\delta_{0}, s_{0}+\delta_{0}\right)$ into $\mathbf{R}$, say $\Phi_{1}, \ldots, \Phi_{k_{0}}$ such that

(a) $\Phi_{j}\left(s_{0}\right)=\sigma_{0}$

(b) $N\left(I-\Phi_{j}(s) A(s)\right) \neq\{0\}$ for $s \in\left(s_{0}-\delta_{0}, s_{0}+\delta_{0}\right), j=1,2, \ldots, k_{0}$;

(c) there exists a neighborhood $V_{0}$ of $\left(\sigma_{0} \cos s_{0}, \sigma_{0} \sin s_{0}\right)$ such that

$\Sigma \cap V_{0}=\left\{\left(\Phi_{j}(s) \cos s, \Phi_{j}(s) \sin s\right): s \in\left(s_{0}-\delta_{0}, s_{0}+\delta_{0}\right), j=1,2, \ldots, k_{0}\right\}$. 
Moreover, for each $j \in\left\{1,2, \ldots, k_{0}\right\}$, there is an analytic function

$$
x_{j}:\left(s_{0}-\delta_{0}, s_{0}+\delta_{0}\right) \rightarrow\left[C_{0}^{1+\alpha}(\bar{\Omega})\right]^{2}
$$

such that $x_{j}(s) \in N\left(I-\Phi_{j}(s) A(s)\right) \backslash\{0\}$ for all $s \in\left(s_{0}-\delta_{0}, s_{0}+\delta_{0}\right)$.

Proof. Lemma 2.2 guarantees that $N\left(I-\sigma_{0} A\left(s_{0}\right)\right)=N\left(\left(I-\sigma_{0} A\left(s_{0}\right)\right)^{2}\right)$, while Lemma 2.1 guarantees that the spectrum of $A(s) \subseteq \mathbf{R}$ for $s \in \mathbf{R}$. Using these facts, the proof is obtained by adapting the proof of $[15$, Chapter $2, \S 2$, Theorem 1 , pp. 57-64] to a Banach space setting, as was done in [4].

Theorem 2.3 has the following corollary which shall prove useful in the sequel.

COROLLARY 2.4. The number of components of $\Sigma$ in a sufficiently small deleted neighborhood of $\left(\sigma_{0} \cos s_{0}, \sigma_{0} \sin s_{0}\right)$ is even and $\leq 2 k_{0}$.

PROOF. Since the functions $\Phi_{1}, \Phi_{2}, \ldots, \Phi_{k_{0}}$ are analytic, if two of them coincide on a subset of $\left(s_{0}-\delta, s_{0}+\delta\right)$ containing a cluster point, they must coincide on all of $\left(s_{0}-\delta, s_{0}+\delta\right)$. Thus, if two of them coincide on $\left(s_{0}-\delta, 0\right)$ they must also coincide on $\left(s_{0}-\delta, s_{0}+\delta\right)$. Hence, the number of arcs corresponding to $s<s_{0}$ is equal to that for $s>s_{0}$, so the total number is even, and clearly less than or equal to $2 k_{0}$.

In the next result we dispense with the symmetry assumption (iv). Without (iv), eigenvalues for (1.1) need not have equal geometric and algebraic multiplicities (e.g., Example (3.9) in [8]). However, if the point $\left(0, \mu_{0}\right), \mu_{0} \in \mathbf{R}$, is a simple eigenvalue for $(1.1)$, we can still give a description of $\Sigma$ near $\left(0, \mu_{0}\right)$. We shall also have use for the next result when we investigate quantitative properties of $\Sigma$.

THEOREM 2.5. Suppose that (i) and (ii) are satisfied, $a_{22}(x)>0$, and $\mu_{0}$ is a simple eigenvalue of

$$
L_{2} \psi=\mu a_{22} \psi \quad \text { in } \Omega, \quad \psi=0 \quad \text { on } \partial \Omega
$$

with eigenfunction $\psi_{0}$ normalized so that $\int_{\Omega} \psi_{0}^{2}=1$. Then in a neighborhood of $\left(0, \mu_{0}\right), \Sigma$ consists of a curve which may be parameterized as $(\lambda, \mu(\lambda)), \lambda \in\left(-\delta_{0}, \delta_{0}\right)$ for some $\delta_{0}>0$, with $\mu(\lambda)$ real analytic in $\lambda$ and $\mu(0)=\mu_{0}$. Corresponding solutions to (1.1) may be expressed as $(u, v, \lambda, \mu)=(\Phi(\lambda), \psi(\lambda), \lambda, \mu(\lambda))$ with $\Phi(\lambda), \psi(\lambda)$ also analytic in $\lambda$.

Proof. We set $X=\left[C_{0}^{2+\alpha}(\bar{\Omega})\right]^{2} \times \mathbf{R}^{2}$ and $Y=\left[C^{\alpha}(\bar{\Omega})\right]^{2} \times \mathbf{R}$ and apply the implicit function theorem to $F: X \rightarrow Y$ defined by

$$
\begin{aligned}
& F(\Phi, \psi, \lambda, \mu) \\
& \quad=\left(L_{1} \Phi-\lambda\left[a_{11} \Phi+a_{12} \psi\right], L_{2} \psi-\mu\left[a_{21} \Phi+a_{22} \psi\right], \int_{\Omega}\left[\Phi^{2}+\psi^{2}\right] d x-1\right) .
\end{aligned}
$$

The real part of the generalized spectrum of (1.1) consists precisely of the points $(\lambda, \mu)$ for which there exist $\Phi, \psi$ such that $F(\Phi, \psi, \lambda, \mu)=(0,0,0)$. It is clear that $F\left(0, \psi_{0}, 0, \mu_{0}\right)=(0,0,0)$, so that $\left(0, \mu_{0}\right)$ belongs to the generalized spectrum. Denote by DF the derivative of $F$ with respect to the variables $\Phi, \psi$, and $\mu$; then for $(p, q, \sigma) \in\left[C_{0}^{2+\alpha}(\bar{\Omega})\right]^{2} \times \mathbf{R}$ we have

$$
\operatorname{DF}\left(0, \psi_{0}, 0, \mu_{0}\right)(p, q, \sigma)=\left(L_{1} p, L_{2} q-\mu_{0}\left(a_{21} p+a_{22} q\right)-\sigma a_{22} \psi_{0}, 2 \int_{\Omega} \psi_{0} q\right) .
$$


If $\operatorname{DF}\left(0, \psi_{0}, 0, \mu_{0}\right)$ is a bijection, then by the open mapping theorem it is a linear homeomorphism, and we may apply the implicit function theorem. To see that the map is injective, suppose that $\operatorname{DF}\left(0, \psi_{0}, 0, \mu_{0}\right)(p, q, \sigma)=(0,0,0)$. We have $L_{1} p=0$ so that $p=0$; thus $L_{2} q=\mu_{0} a_{22} q+\sigma a_{22} \psi_{0}$. Multiplying the last equation by $\psi_{0}$, integrating by parts over $\Omega$ via the divergence theorem and the selfadjoint structure of $L_{2}$, and using (2.5) yields $\sigma \int_{\Omega} a_{22} \psi_{0}^{2}=0$. Hence, $\sigma=0$; but then $L_{2} q-\mu_{0} a_{22} q=0$, and we also have $2 \int_{\Omega} q \psi_{0}=0$. Since $\mu_{0}$ is a simple eigenvalue of (2.5) with eigenspace $\left\langle\psi_{0}\right\rangle, \rho=0$ is a simple eigenvalue of $\left(L_{2}-\mu_{0} a_{22}\right) \psi=\rho \psi$ in $\Omega, \psi=0$ on $\partial \Omega$, also with eigenspace $\left\langle\psi_{0}\right\rangle$, so since $\int_{\Omega} q \psi_{0}=0$, we must have $q=0$. Thus, $\mathrm{DF}\left(0, \psi_{0}, 0, \mu_{0}\right)$ is injective. To show the surjectivity of $\mathrm{DF}\left(0, \psi_{0}, 0, \mu_{0}\right)$, we try solving $\operatorname{DF}\left(0, \psi_{0}, 0, \mu_{0}\right)(p, q, \sigma)=(f, g, \tau) \in Y$. For the first component we must solve $L_{1} p=f$ in $C_{0}^{2+\alpha}(\bar{\Omega})$, which is possible by our assumptions on $L_{1}$. Hence, $p$ is determined, and for the second component we must solve

$$
L_{2} q-\mu_{0} a_{22} q=g+\mu_{0} a_{21} p+\sigma a_{22} \psi_{0}
$$

for $q$ in $C_{0}^{2+\alpha}(\bar{\Omega})$. By the Fredholm alternative we can solve (2.6) provided that $\sigma$ is chosen so that

$$
\sigma \int_{\Omega} a_{22} \psi_{0}^{2}=-\int_{\Omega} \psi_{0}\left[\left(g+\mu_{0} a_{21} p\right)\right]
$$

Making that choice of $\sigma$, we solve (2.6). The solution will not be unique; if $\tilde{q}$ is a solution, so is $\tilde{q}+s \psi_{0}$ for any $s \in \mathbf{R}$. Fix $\tilde{q}$; then for the third component we must solve

$$
2 \int_{\Omega} \psi_{0} q=2 \int_{\Omega} \psi_{0}\left(\tilde{q}+s \psi_{0}\right)=\tau
$$

However, (2.7) is equivalent to

$$
2 s \int_{\Omega} \psi_{0}^{2}=\tau-2 \int_{\Omega} \psi_{0} \tilde{q},
$$

which we can satisfy by making the appropriate choice of $s$. That choice determines $q$ and gives our solution; hence $\operatorname{DF}\left(0, \psi_{0}, 0, \mu_{0}\right)$ is surjective. Hence, we may apply the implicit function theorem to assert that for $\lambda$ in some interval $\left(-\delta_{0}, \delta_{0}\right)$, there are functions $\Phi(\lambda), \psi(\lambda), \mu(\lambda)$ with $\Phi(0)=0, \psi(0)=\psi_{0}$, and $\mu(0)=\mu_{0}$ satisfying $F(\Phi(\lambda), \psi(\lambda), \lambda, \mu(\lambda))=(0,0,0)$, which implies that $(\lambda, \mu(\lambda))$ belongs to the generalized spectrum. To conclude that the dependence on $\lambda$ is analytic, we note that we may complexify $X$ and $Y$ and that for fixed $\Phi_{j}, \psi_{j}, \lambda_{j}, \mu_{j}, j=1,2$, the function $F\left(\Phi_{1}+z_{1} \Phi_{2}, \psi_{1}+z_{2} \psi_{2}, \lambda_{1}+z_{3} \lambda_{2}, \mu_{1}+z_{4} \mu_{2}\right)$ is complex analytic in $z_{1}, z_{2}, z_{3}, z_{4}$. It follows from the analytic version of the implicit function theorem (see [16, Theorem 1.48]) that $\Phi(\lambda), \psi(\lambda)$ and $\mu(\lambda)$ are analytic in $\lambda$ near $\lambda=0$. We have already shown that $\Phi(\lambda), \psi(\lambda)$, and $\mu(\lambda)$ are real for $\lambda$ real, so we have real analyticity for $\lambda \in\left(-\delta_{0}, \delta_{0}\right)$ with $\delta_{0}$ sufficiently small.

REMARK. Theorem 2.5 was partially motivated by some ideas used in [6]. The corresponding result clearly holds near $\left(\lambda_{0}, 0\right)$ if $\lambda_{0}$ is a simple eigenvalue for

$$
L_{1} \Phi=\lambda a_{11} \Phi \text { in } \Omega, \quad \Phi=0 \text { on } \partial \Omega .
$$

3. Quantitative results. We now turn to some quantitative questions about $\Sigma$. Our first result describes how arcs in $\Sigma$ emanate from points $\left(0, \mu_{0}\right)$ or $\left(\lambda_{0}, 0\right)$ where $\mu_{0}$ and $\lambda_{0}$ are simple eigenvalues of (2.5) and (2.8) respectively. For convenience we state the result for (2.5). 
THEOREM 3.1. Suppose that (i)-(iv) hold and that $\mu_{0}$ is a simple eigenvalue for (2.5) with corresponding normalized eigenfunction $\psi_{0}$. If $(\Phi(\lambda), \psi(\lambda), \lambda, \mu(\lambda))$ is as in Theorem 2.5, we have

$$
\mu^{\prime}(0) \leq 0,
$$

with strict inequality if $a_{12}(x) \psi_{0}(x) \not \equiv 0$.

The expressions $\mu^{\prime}(0)$ and $\Phi^{\prime}(0)$ can in principle be calculated; see (3.2), (3.6) below.

ProOF. By Theorem 2.5, we may differentiate (1.1) with respect to $\lambda$ at $\lambda=0$. Doing so, denoting $\Phi^{\prime}(0)$ by $\Phi_{1}$, and substituting $(\Phi(0), \psi(0), 0, \mu(0))=$ $\left(0, \psi_{0}, 0, \mu_{0}\right)$ yields

$$
L_{1} \Phi_{1}=a_{12} \psi_{0}
$$

with $\Phi_{1} \in C_{0}^{2+\alpha}(\bar{\Omega})$. The analyticity of $\Phi(\lambda)$ and $\psi(\lambda)$ then permits us to write $\Phi(\lambda)=\lambda \Phi_{1}+\lambda^{2} \Phi_{2}(\lambda), \psi(\lambda)=\psi_{0}+\lambda \psi_{1}(\lambda)$, with $\Phi_{2}$ and $\psi_{1}$ bounded as $\lambda \rightarrow 0$. Using that representation in (1.1), multiplying the second equation by $\psi_{0}$, then integrating yields

$$
\begin{aligned}
& \int_{\Omega} \psi_{0}\left(L_{2} \psi_{0}+\lambda L_{2} \psi_{1}\right) \\
& \quad=\mu(\lambda) \int_{\Omega}\left[\lambda a_{12} \Phi_{1} \psi_{0}+\lambda^{2} a_{12} \Phi_{2} \psi_{0}+a_{22} \psi_{0}^{2}+\lambda a_{22} \psi_{0} \psi_{1}\right] .
\end{aligned}
$$

Using (2.5) and integration by parts in the integral on the left side of (3.3) yields

$$
\begin{aligned}
& \mu(0) \int_{\Omega}\left(a_{22} \psi_{0}^{2}+\lambda a_{22} \psi_{0} \psi_{1}\right) \\
& \quad=\mu(\lambda) \int_{\Omega}\left[a_{22} \psi_{0}^{2}+\lambda a_{22} \psi_{0} \psi_{1}\right]+\lambda \mu(\lambda) \int_{\Omega} a_{12} \Phi_{1} \psi_{0}+\lambda^{2} \mu(\lambda) \int_{\Omega} a_{12} \Phi_{2} \psi_{0}
\end{aligned}
$$

By (3.2) we may replace $a_{12} \psi_{0}$ with $L_{1} \Phi_{1}$ in the second integral on the right side of (3.4). Rearranging (3.4) then yields

$$
\begin{aligned}
{[\mu(\lambda)} & -\mu(0)] \int_{\Omega}\left[a_{22} \psi_{0}^{2}+\lambda a_{22} \psi_{0} \psi_{1}\right] \\
& =-\lambda \mu(\lambda)\left[\int_{\Omega} \Phi_{1} L_{1} \Phi_{1}+\lambda \int_{\Omega} a_{12} \Phi_{2} \psi_{0}\right] .
\end{aligned}
$$

For $\lambda$ near 0 , the integral on the left side of (3.5) is positive, so we have

$$
\frac{\mu(\lambda)-\mu(0)}{\lambda}=-\mu(\lambda) \frac{\int_{\Omega} \Phi_{1} L_{1} \Phi_{1}+\lambda \int_{\Omega} a_{12} \Phi_{2} \psi_{0}}{\int_{\Omega}\left(a_{22} \psi_{0}^{2}+\lambda a_{22} \psi_{0} \psi_{1}\right)} .
$$

Letting $\lambda \rightarrow 0$, we have

$$
\mu^{\prime}(0)=-\mu_{0} \int_{\Omega} \Phi_{1} L_{1} \Phi_{1} / \int_{\Omega} a_{22} \psi_{0}^{2} .
$$

By (i)-(iii) we have $\mu_{0}>0$ and the integral in the numerator in (3.6) nonnegative. That integral will be strictly positive if $\Phi_{1} \not \equiv 0$, which will be true by (3.2) if $a_{12} \psi_{0} \not \equiv 0$. 
REMARK. Theorems 2.5 and 3.1 are quantitative in the sense that they give information about the location and direction of some of the arcs constituting $\Sigma$ in terms of quantities which can in principle be calculated. The computational nature of the result depends on the hypothesis that $\mu_{0}$ is a simple eigenvalue, which has the consequence of allowing us to use $\lambda$ instead of arclength as a parameter determining $\Phi, \psi$, and $\mu$. In the more general case it may be possible to use the parameterization of Theorem 2.3 and obtain some information about $d \lambda / d s$ and $d \mu / d s$ near a point $s_{0}$ where we have some information about $\lambda\left(s_{0}\right), \mu\left(s_{0}\right)$, and the corresponding eigenspace for (1.1).

We shall now employ a variational characterization of eigenvalues to describe how points of $\Sigma$ are distributed along rays in the first quadrant in $\mathbf{R}^{2}$. To formulate the variational approach, we consider systems of the form

$$
\begin{gathered}
L_{i} w_{i}=\sigma \sum_{j=1}^{2} a_{i j}(x) w_{j} \quad \text { in } \Omega, \quad i=1,2, \\
w_{1}=w_{2}=0 \quad \text { on } \partial \Omega
\end{gathered}
$$

where the operators $L_{i}$ satisfy (i) and the coefficients $a_{i j}$ satisfy (ii)-(iv) so that $\left(a_{i j}\right)_{i, j=1}^{2}$ is a positive definite symmetric matrix. We observe that for such systems we may apply the variational formulation of the eigenvalue problem given by Courant and Hilbert [9]. By the type of analysis done in Lemma 2.1, we see that spectrum of (3.7) consists of a discrete set $\left\{\sigma_{k}\right\}$ of real eigenvalues which can be ordered so that $\sigma_{k} \leq \sigma_{k+1}$ and $\sigma_{k} \rightarrow \infty$ as $k \rightarrow \infty$. Following Courant and Hilbert, we set

$$
D^{l}[\Phi]=\int_{\Omega}\left[\sum_{i, j=1}^{n} A_{i j}^{l}(x) \Phi_{x_{i}} \Phi_{x_{j}}+A^{l}(x) \Phi^{2}\right], \quad l=1,2,
$$

and

$$
H[(u, v),(\Phi, \psi)]=\int_{\Omega}\left[a_{11} u \Phi+a_{12}(u \psi+v \Phi)+a_{22} v \psi\right]
$$

with $H[(u, v)] \equiv H[(u, v),(u, v)]$. We observe that by (ii)-(iv) $H$ defines an inner product, and write $(\Phi, \psi)^{\perp}(u, v)$ if $H[(\Phi, \psi),(u, v)]=0$. It follows precisely as in the case of single equation treated in [9] that the $k$ th eigenvalue for (3.7) is given by

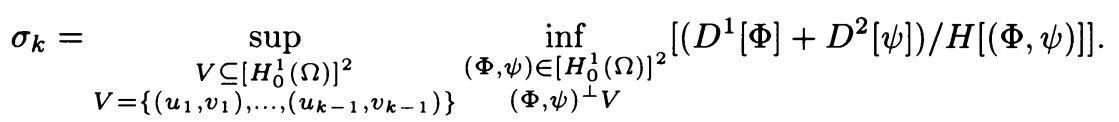

The characterization (3.10) yields a comparison theorem for eigenvalues of (3.7) analogous to those given for a single equation in [9, Chapter VI, §1]. We state the comparison result below, but omit the proof; it is essentially the same as that given by Courant and Hilbert for a single equation.

THEOREM 3.2. Suppose that $\left\{\sigma_{k}^{1}\right\},\left\{\sigma_{k}^{2}\right\}$ are the spectra for two systems of the form (3.7), with $D_{\alpha}^{l}, H_{\alpha}, \alpha=1,2$, the corresponding forms defined in (3.8), (3.9). If $D_{1}^{l}[u] \geq D_{2}^{l}[u]$ for $l=1,2$ and all $u \in H_{0}^{1}(\Omega)$ and $H_{1}[(u, v)] \leq H_{2}[(u, v)]$ for all $(u, v) \in\left[H_{0}^{1}(\Omega)\right]^{2}$, then for all $k$ we have $\sigma_{k}^{1} \geq \sigma_{k}^{2}$. 
Theorem 3.2 is quantitative in the sense that it allows us to obtain information about the location of points of $\Sigma$ for (1.1) via comparison with other systems. We will use that approach to obtain most of our remaining results.

To apply Theorem 3.2 we must recast (1.1) in the form (3.7). To that end, we consider the points of the generalized spectrum lying along a fixed ray in the real $\lambda-\mu$ plane. For any $m>0$ we consider the ray $\mu=\lambda m$; along that ray, (1.1) may be written as

$$
L_{1} \Phi=\lambda\left(a_{11} \Phi+a_{12} \psi\right), \quad(1 / m) L_{2} \psi=\lambda\left(a_{12} \Phi+a_{22} \psi\right)
$$

or, equivalently,

$$
m L_{1} \Phi=\mu\left(a_{11} \Phi+a_{12} \psi\right), \quad L_{2} \psi=\mu\left(a_{12} \Phi+a_{22} \psi\right) .
$$

Both (3.11) and (3.12) have the same form as (3.7). The points of $\Sigma$ lying on the ray $\mu=\lambda m$ can thus be characterized as $\left(\lambda_{k}(m), \mu_{k}(m)\right)$ with $\mu_{k}(m)=m \lambda_{k}(m)$ and with $\lambda_{k}(m), \mu_{k}(m)$ the $k$ th eigenvalues for (3.11) and (3.12) respectively. Hence, we can use Theorem 3.2 to describe the direction of the $\operatorname{arcs}$ constituting $\Sigma$ in the first quadrant. We have

THEOREM 3.3. Suppose (i)-(iv) hold. If for $m>0\left(\lambda_{k}(m), \mu_{k}(m)\right)$ denotes the $k$ th point of intersection (counting multiplicities) of $\Sigma$ with the ray $\mu=\lambda m, \lambda>0$, then, $\left(\lambda_{k}(m), \mu_{k}(m)\right)$ varies continuously with $m$. As functions of $m, \lambda_{k}(m)$ is nonincreasing; and $\mu_{k}(m)=m \lambda_{k}(m)$ is nondecreasing.

REMARKS. It follows from Theorem 3.3 that as $(\lambda, \mu)$ moves along one of the arcs constituting the set $\Sigma$ in the direction of increasing $\lambda, \mu$ must be nonincreasing. If $(\lambda, \mu)$ passes through a point of the generalized spectrum with multiplicity greater than one, then in general several arcs will emanate from the point; however, all of them must be nonincreasing in $\mu$ as $\lambda$ increases. Because of this phenomenon, the curve $\left(\lambda_{k}(m), \mu_{k}(m)\right)$ will generally not be differentiable in $m$. That such is the case may be seen by considering the constant coefficient problem studied in [5].

ProOF OF THEOREM 3.3. Defining $D^{\alpha}$ as in (3.8) and taking $H$ as defined by (3.9) for the systems (3.11) and (3.12), we have by (3.10) applied to (3.11) that

$$
\lambda_{k}(m)=\sup _{\substack{V \subseteq\left[H_{0}^{1}(\Omega)\right]^{2} \\ V=\left\{\left(u_{1}, v_{1}\right), \ldots,\left(u_{k-1}, v_{k-1}\right)\right\}}} \inf _{\substack{(\Phi, \psi) \in\left[H_{0}^{1}(\Omega)\right]^{2} \\(\Phi, \psi)^{\perp} V}} \frac{D^{1}[\Phi]+(1 / m) D^{2}[\psi]}{H[(\Phi, \psi)]} .
$$

Clearly, $(1 / m) D^{2}[\psi]$ is nonincreasing for any $\psi$ as $m$ increases; $D^{1}$ and $H$ are unchanged, so by Theorem $3.2 \lambda_{k}(m)$ is nonincreasing in $m$. To see that $\mu_{k}(m)$ is nondecreasing in $m$, we apply (3.10) to (3.12) to obtain

$$
\mu_{k}(m)=\sup _{\substack{V \subseteq\left[H_{0}^{1}(\Omega)\right]^{2} \\ V=\left\{\left(u_{1}, v_{1}\right), \ldots,\left(u_{k-1}, v_{k-1}\right)\right\}}} \inf _{\substack{(\Phi, \psi) \in\left[H_{0}^{1}(\Omega)\right]^{2} \\(\Phi, \psi)^{\perp} V}} \frac{m D^{1}[\Phi]+D^{2}[\psi]}{H[(\Phi, \psi)]}
$$

and note that $m D^{1}[\Phi]$ is nondecreasing in $m$ and $D^{2}$ and $H$ do not involve $m$, and again apply Theorem 3.2.

Notice now that if $\delta>0$ is such that $m-\delta>0$, we have

$$
\lambda_{k}(m+\delta) \leq \lambda_{k}(m) \leq \lambda_{k}(m-\delta)
$$


and

$$
\mu_{k}(m+\delta) \geq \mu_{k}(m) \geq \mu_{k}(m-\delta)
$$

Since (3.16) is equivalent to

$$
(m+\delta) \lambda_{k}(m+\delta) \geq m \lambda_{k}(m) \geq(m-\delta) \lambda_{k}(m-\delta),
$$

(3.15) and (3.16) imply that

$$
\frac{m}{m+\delta} \lambda_{k}(m) \leq \lambda_{k}(m+\delta) \leq \lambda_{k}(m) \leq \lambda_{k}(m-\delta) \leq \frac{m}{m-\delta} \lambda_{k}(m) .
$$

Therefore $\lambda_{k}(m)$ and $\mu_{k}(m)=m \lambda_{k}(m)$ are continuous functions of $m$.

The final result of this section tells how points $(0, \mu)$ can be connected to points $(\lambda, 0)$ via $\operatorname{arcs}$ in $\Sigma$.

THEOREM 3.4. Suppose that (i)-(iv) hold. Let $\mu_{k}$ denote the $k$ th eigenvalue of (2.5) and $\lambda_{k}$ the kth eigenvalue of (2.8). Then there exists an arc in $\Sigma$ connecting $\left(0, \mu_{k}\right)$ to $\left(\lambda_{k, 0}\right)$.

ProOF. We can write (1.1) as

$$
L_{1} u=\sigma \cos s\left(a_{11} u+a_{12} v\right), \quad L_{2} v=\sigma \sin s\left(a_{12} u+a_{22} v\right)
$$

which is equivalent to (3.12) with $\mu=\sigma \sin s$ and $m=\tan s, s \in(0, \pi / 2)$. If we start at $s=\pi / 2$ and let $s$ decrease toward zero, then by Theorem 2.3 the part of $\Sigma$ in the first quadrant lying in a neighborhood of $\left(0, \mu^{*}\right)$ with $\mu^{*}=\mu_{k}$ consists of a system of analytic arcs equal in number to the multiplicity of $\mu^{*}$. By fixing $k$ and choosing $s$ close enough to $\pi / 2$, we can put the arcs into oneto-one correspondence with $\mu_{k}, \mu_{k+1}, \ldots, \mu_{k+l-1}$, where $l$ is the multiplicity of $\mu^{*}$. Choosing the arc corresponding to $\mu_{k}$, we see that for some $\varepsilon>0$, if $s=\pi / 2-\varepsilon$, the point where the $k$ th arc meets the ray $\mu=m \lambda$ with $m=\tan s$ is $\left(\lambda_{k}(m), \mu_{k}(m)\right)$ where $\left(\lambda_{k}(m), \mu_{k}(m)\right)$ is as in Theorem 3.3. Then by Theorem 3.3 we can decrease $s$ and hence $m$ until $\left(\lambda_{k}(m), \mu_{k}(m)\right)$ meets the arc corresponding to $\lambda_{k}$ and emanating from $\left(\lambda^{*}, 0\right)$ with $\lambda^{*}=\lambda_{k}$. (That such an arc exists and meets $\left(\lambda_{k}(m), \mu_{k}(m)\right)$ for $m$ sufficiently small follows from the same argument used to assert the existence of such an arc emanating from $\left(0, \mu^{*}\right)$ with $\mu^{*}=\mu_{k}$.)

4. Multiplicity estimates. We now consider the problem of bounding the dimension of the eigenspace at points of the generalized spectrum. Our results are qualitative in content but require some of the quantitative methods of the previous section. In general, there will be points at which the eigenspace will have dimension two. As shown in [5], this phenomenon already occurs in the case where $L^{1}=L^{2}=L$, all the eigenvalues for $L \Phi=\lambda \Phi$ in $\Omega, \Phi=0$ on $\partial \Omega$, are simple, and the coefficients $a_{i j}$ are constant. The issue is complicated further by the fact that question of multiplicities of eigenvalues for a single elliptic operator is a subtle and difficult one. Methods based on the theory of positive operators can be used to show that under appropriate hypotheses the first eigenvalue of a second-order elliptic system is simple; see $[\mathbf{7}, \mathbf{8}]$. Even for the Laplacian, higher eigenvalues may have multiplicity greater than one. However, the simplicity of all eigenvalues of the Laplacian with Dirichlet boundary conditions on domains in $\mathbf{R}^{n}$ is known to be generic (in an appropriate sense) with respect to the domain; see $[\mathbf{1 2}, \mathbf{1 7}]$. We shall proceed by assuming that $\Omega$ is a domain for which the eigenvalues of 
the Laplacian are all simple and using Theorem 3.2 to compare our system with a simpler one, constructed from the Laplacian, for which we can readily determine the eigenvalues occurring along the ray $\mu=\lambda m$. Since the choice of comparison systems is somewhat arbitrary, our approach illustrates a situation common in the theory of partial differential equations: the technique is more significant than any specific result that it yields. Thus our analysis is not the only possible one, but serves to explicate the method. (The question of estimating the first eigenvalue is somewhat different than that of estimating higher eigenvalues; that problem was treated by Protter in [14].)

In the remainder of this section we shall assume the following:

HyPOTHESIS S. The domain $\Omega$ is such that the spectrum of

$$
-\Delta w=\gamma w \quad \text { in } \Omega, \quad w=0 \quad \text { on } \partial \Omega
$$

consists of a sequence $\gamma_{1}<\gamma_{2}<\gamma_{3}<\cdots$ of simple eigenvalues. As noted above, Hypothesis $\mathrm{S}$ is satisfied generically with respect to $\Omega$.

We shall proceed by considering points of $\Sigma$ lying on the ray $\mu=m \lambda, \lambda>0$, and comparing them with multiples of the eigenvalues for (4.1) via Theorem 3.2. Following the notation of Theorem 3.3 , let $\left((1 / m) \mu_{k}(m), \mu_{k}(m)\right)$ denote the $k$ th point of intersection of $\Sigma$ with the ray emanating from the origin given by $\mu=$ $m \lambda, \lambda>0$. For specified regions of the $\lambda-\mu$ plane we will show via Theorem 3.2 that for each $m$ there are intervals $I_{k}$ which can be given explicitly in terms of eigenvalues of (4.1) and bounds on coefficients in our system, and for which $\mu_{k}(m) \in I_{k}$. We will then give conditions under which no point on $\mu=m \lambda$ can belong to more than two such intervals and hence no eigenvalue on the ray can have multiplicity greater than two.

To obtain bounds for $\mu_{k}(m)$ via comparison of our system with simpler ones, we must give explicit bounds for certain quantities depending on the coefficients of our system. We shall assume conditions (i)-(iv). Further, we shall assume that for $l=1,2, e_{l}$ and $E_{l}$ are positive constants such that

$$
\begin{gathered}
e_{l}|\xi|^{2} \leq \sum_{i, j=1}^{n} A_{i j}^{l}(x) \xi_{i} \xi_{j} \leq E_{l}|\xi|^{2}, \quad x \in \Omega, \quad \xi \in \mathbf{R}^{n}, \\
A^{l}(x)=0, \quad x \in \Omega .
\end{gathered}
$$

(The condition $A^{l}(x)=0$ is not necessary for our arguments, but simplifies the computations.) If we set $h(u, v)=a_{11}(x) u^{2}+2 a_{12}(x) u v+a_{22}(x) v^{2}$, then we have $\sigma_{-}(x)\left(u^{2}+v^{2}\right) \leq h(u, v) \leq \sigma_{+}(x)\left(u^{2}+v^{2}\right)$ where $\sigma_{-}(x)$ and $\sigma_{+}(x)$ are respectively the smaller and larger eigenvalues of the matrix $\left(\left(a_{i j}(x)\right)\right)$. Since by (iii) the matrix is positive definite in $\bar{\Omega}$, we have

$$
q\left(u^{2}+v^{2}\right) \leq h(u, v) \leq Q\left(u^{2}+v^{2}\right)
$$

where $Q \equiv \sup _{\bar{\Omega}} \sigma_{+}(x) \geq \inf _{\bar{\Omega}} \sigma_{-}(x) \equiv q>0$. Finally, for each fixed $k \in Z^{+}$we have by Hypothesis $\mathrm{S}$ that

$$
R(K) \equiv \inf _{k \in\{1, \ldots, K\}}\left(\delta_{k+1} / \delta_{k}\right)>1
$$

We can now state a multiplicity result based on Theorem 3.2. 
THEOREM 4.1. Suppose that Hypothesis S and conditions (i)-(iv), (4.2) and (4.3) hold. Let $R(K)$ be as defined in (4.4) and suppose that we can choose positive constants $b, B$ with $b<B$ such that

$$
b e_{1}<e_{2}<E_{2}<B E_{1}
$$

and

$$
R(K)>\max \left\{B E_{1} / b e_{1}, E_{2} Q / b e_{1} q, B E_{1} Q / e_{2} q\right\} .
$$

Then if $(\lambda, \mu) \in \Sigma$ is in the region of the $\lambda-\mu$ plane bounded by $\mu=b \lambda, \mu=B \lambda$, and $\mu=E_{2} \gamma_{K} / q$, the dimension of the eigenspace for $(1.1)$ at $(\lambda, \mu)$ is either one or two.

REMARK. Condition 4.6 will be met if for example we can take all the constants on the right sufficiently close to one; or if we are given any set of constants and $R(K)$ is sufficiently large. The condition arises in its specific form from our choice of comparison systems; there are various other possibilities.

PROOF. We consider the comparison systems

$$
\begin{aligned}
-b e_{1} \Delta u=\mu Q u, & \\
-e_{2} \Delta v=\mu Q v & \text { in } \Omega, \\
u=v=0 & \text { on } \partial \Omega
\end{aligned}
$$

and

$$
\begin{array}{rlrl}
-B E_{1} \Delta u & =\mu q u, & \\
-E_{2} \Delta v & =\mu q v & & \text { in } \Omega, \\
u=v & =0 & & \text { on } \partial \Omega,
\end{array}
$$

where the constants are as in (4.2), (4.3). The eigenvalues for (4.7) belong to the sequences $\left(\underline{\mu}_{j}^{1}\right)_{j \in Z^{+}} \equiv\left(\left(b e_{1} / Q\right) \gamma_{j}\right)_{j \in Z^{+}}$and $\left(\underline{\mu}_{j}^{2}\right)_{j \in Z^{+}} \equiv\left(\left(e_{2} / Q\right) \gamma_{j}\right)_{j \in Z^{+}} ;$similarly, the eigenvalues for (4.8) belong to the sequences $\left(\bar{\mu}_{j}^{1}\right)_{j \in Z^{+}} \equiv\left(\left(B E_{1} / q\right) \gamma_{j}\right)_{j \in Z^{+}}$and $\left(\bar{\mu}_{j}^{2}\right)_{j \in Z^{+}} \equiv\left(\left(E_{2} / q\right) \gamma_{j}\right)_{j \in Z^{+}}$. It is not immediately obvious how the full spectra $\left(\underline{\mu}_{k}\right)_{k \in Z^{+}}$and $\left(\bar{\mu}^{k}\right)_{k \in Z^{+}}$of (4.7) and (4.8) respectively are related to the sequences $\left(\underline{\mu}_{j}^{1}\right),\left(\underline{\mu}_{j}^{2}\right),\left(\bar{\mu}_{j}^{1}\right)$, and $\left(\bar{\mu}_{j}^{2}\right)$. However, by $(4.5)$ and (4.6) we have

$$
b e_{1}<e_{2}<E_{2} Q / q<R(K) b e_{1}
$$

and similarly

$$
E_{2}<B E_{1}<E_{2} R(K) .
$$

(For our purposes inequalities of the form $e_{2}<b e_{1}<R(K) e_{2}$ and/or $B E_{1}<E_{2}<$ $B E_{1} R(K)$ would serve as well as (4.9), (4.10); using those inequalities instead of (4.9), (4.10) would lead us to require a slightly different form of (4.6).) It follows from (4.9), (4.10) that $\underline{\mu}_{j}^{1}<\underline{\mu}_{j}^{2}<\underline{\mu}_{j+1}^{1}$ and $\bar{\mu}_{j}^{2}<\bar{\mu}_{j}^{1}<\bar{\mu}_{j+1}^{2}$ for $1 \leq j \leq K$; thus for $1 \leq i \leq K$ we may take $\underline{\mu}_{2 i-1}=\underline{\mu}_{i}^{1}, \underline{\mu}_{2 i}=\underline{\mu}_{i}^{2}, \bar{\mu}_{2 i-1}=\bar{\mu}_{i}^{2}$ and $\bar{\mu}_{2 i}=\bar{\mu}_{i}^{1}$. We now use Theorem 3.2 to compare the eigenvalues of $(4.7),(4.8)$ with those of $(3.12)$. (We recall that $\Sigma$ is determined by those eigenvalues.) Conditions (4.2) and (4.3) permit the comparisons; we have for example

$$
H[(u, v)] \equiv \int h(u, v) \leq Q \int\left(u^{2}+v^{2}\right)
$$


and

$$
\begin{gathered}
b e_{1} \int|\nabla u|^{2} \leq m \int \sum_{i, j=1}^{n} A_{i j}^{1} u_{x_{i}} u_{x_{j}} \equiv m D^{1}[u] \\
e_{2} \int|\nabla v|^{2} \leq \int \sum_{i, j=1}^{n} A_{i j}^{2} v_{x_{i}} v_{x_{j}} \equiv D^{2}[v]
\end{gathered}
$$

so that $\underline{\mu}_{k} \leq \mu_{k}(m)$; similarly $\mu_{k}(m) \leq \bar{\mu}^{k}$, provided $b<m<B$. Thus, we have for $1 \leq \bar{k} \leq K$

$$
\begin{aligned}
\left(b e_{1} / Q\right) \gamma_{k} & \leq \mu_{2 k-1}(m) \leq\left(E_{2} / q\right) \gamma_{k}, \\
\left(e_{2} / Q\right) \gamma_{k} & \leq \mu_{2 k}(m) \leq\left(B E_{1} / q\right) \gamma_{k} .
\end{aligned}
$$

The inequalities (4.11) establish intervals in which the eigenvalues $\mu_{k}$ must lie. To see that no eigenvalue can have multiplicity greater than two, it suffices to observe that there are no points belonging to more than two such intervals. Another way of expressing that information is via the following inequalities, obtained from (4.6) and (4.11):

$$
\begin{aligned}
\bar{\mu}_{2 j-1} & =\bar{\mu}_{j}^{2}=\left(E_{2} / q\right) \gamma_{j}<\left(b e_{1} R(K) / Q\right) \gamma_{j} \\
& \leq\left(b e_{1} / Q\right) \gamma_{j+1}=\underline{\mu}_{j+1}^{1}=\underline{\mu}_{2 j+1}
\end{aligned}
$$

and

$$
\bar{\mu}_{2 j}=\bar{\mu}_{j}^{1}=\left(B E_{1} / q\right) \gamma_{j}<\left(e_{2} R(K) / Q\right) \gamma_{j} \leq \underline{\mu}_{j+1}^{2}=\underline{\mu}_{2 j+2} .
$$

Hence $\bar{\mu}_{k}<\underline{\mu}_{k+2}$, so that no point can belong to more than two intervals $I_{k}=$ $\left[\underline{\mu}_{k}, \bar{\mu}_{k}\right]$. Thus, no eigenvalue $\mu_{k}(m)$ can have multiplicity greater than two. Since the points of $\Sigma$ have the form $\left((1 / m) \mu_{k}(m), \mu_{k}(m)\right)$, the dimension of the nullspace for (1.1) is at most two.

5. Asymptotic results. We have seen that for $\lambda$ near zero, $\Sigma$ consists of arcs emanating from the point $(0, \mu)$ where $\mu$ is an eigenvalue for $L_{2} \psi=\mu a_{22} \psi$ in $\Omega, \psi=0$ on $\partial \Omega$. We now consider the behavior of $\Sigma$ for $\lambda$ large and positive. We shall assume that conditions (i)-(iv) are satisfied, and further that (4.2) holds and $a_{11}, a_{12} \in C^{1}(\bar{\Omega})$. Let $\gamma_{k}(L, c(x))$ denote the $k$ th eigenvalue for

$$
L w=\gamma c(x) w \quad \text { in } \Omega, \quad w=0 \quad \text { on } \partial \Omega .
$$

Define $a_{\infty}(x)$ by

$$
a_{\infty}(x) \equiv a_{22}-a_{12}^{2} / a_{11}
$$

We then have the following:

THEOREM 5.1. For each $\delta>0$ and $j \in Z^{+}$, there exists a number $\bar{\lambda}$ such that $\Sigma$ has a subset $S_{j} \subseteq\left\{(\lambda, \mu): \lambda>\bar{\lambda},\left|\mu-\gamma_{j}\left(L_{2}, a_{\infty}\right)\right|<\delta\right\}$ with $S_{j} \cap[\{\lambda\} \times\{\mu$ : $\left.\left.\left|\mu-\gamma_{j}\left(L_{2}, a_{\infty}\right)\right|<\delta\right\}\right]$ is nonempty for all $\lambda>\bar{\lambda}$.

REMARK. Since $\Sigma$ consists of a network of piecewise analytic arcs, we can thus vary $(\lambda, \mu)$ continuously in such a way that $(\lambda, \mu) \in \Sigma, \lambda \rightarrow \infty$, and $\mu \rightarrow \gamma_{j}\left(L_{2}, a_{\infty}\right)$.

ProOF. Let $D^{1}, D^{2}$, and $H$ be as defined in (3.8), (3.9). Let $P$ be the matrix

$$
P=\left(\begin{array}{cc}
1 & a_{12} / a_{11} \\
0 & 1
\end{array}\right) ;
$$


then

$$
P^{-1}=\left(\begin{array}{cc}
1 & -a_{12} / a_{11} \\
0 & 1
\end{array}\right)
$$

We note that multiplication of a vector valued function (written as a column vector) by $P$ generates a bounded, invertible operator on $\left[H_{0}^{1}(\Omega)\right]^{2}$. Let

$$
\tilde{H}[(u, v),(\Phi, \psi)]=\int\left(a_{11} u \Phi+a_{\infty} v \psi\right) .
$$

A calculation shows that if $\mathbf{w}_{1}, \mathbf{w}_{2} \in\left[H_{0}^{1}(\Omega)\right]^{2}$, then

$$
\tilde{H}\left[P \mathbf{w}_{1}, P \mathbf{w}_{2}\right]=H\left[\mathbf{w}_{1}, \mathbf{w}_{2}\right] .
$$

We shall write $\mathbf{w}_{1} \perp_{H} \mathbf{w}_{2}$ if $H\left[\mathbf{w}_{1}, \mathbf{w}_{2}\right]=0$ and $\mathbf{w}_{1} \perp_{\tilde{H}} \mathbf{w}_{2}$ if $\tilde{H}\left[\mathbf{w}_{1}, \mathbf{w}_{2}\right]=0$. By (5.4) we see that $\mathbf{w}_{1} \perp_{H} \mathbf{w}_{2}$ if and only if $P \mathbf{w}_{1} \perp_{\tilde{H}} P \mathbf{w}_{2}$. If $V=\left\{\mathbf{w}_{1}, \ldots, \mathbf{w}_{k-1}\right\}$ and $P V=\left\{P \mathbf{w}_{1}, \ldots, P \mathbf{w}_{k-1}\right\}$ then

$$
\mathbf{w} \perp_{H} V \text { if and only if } P \mathbf{w} \perp_{\tilde{H}} P V \text {. }
$$

If we denote $\mathbf{w}$ by $(u, v)$ and $P \mathbf{w}$ by $(\tilde{u}, \tilde{v})$ then $\tilde{v}=v$ and $\tilde{u}=u+a_{12} v / a_{11}$ so that $u=\tilde{u}-a_{12} \tilde{v} / a_{11}$. Hence $D^{1}[u]=D^{1}\left[\tilde{u}-a_{12} \tilde{v} / a_{11}\right]$ and $D^{2}[v]=D^{2}[\tilde{v}]$. Thus we have

$$
\frac{m D^{1}[u]+D^{2}[v]}{H[(u, v)]}=\frac{m D^{1}\left[\tilde{u}-a_{12} \tilde{v} / a_{11}\right]+D^{2}[\tilde{v}]}{\tilde{H}[(\tilde{u}, \tilde{v})]}
$$

and by (5.5)

$$
\inf _{(u, v) \perp_{H} V} \frac{m D^{1}[u]+D^{2}[v]}{H[(u, v)]}=\inf _{(\tilde{u}, \tilde{v}) \perp_{\tilde{H}} P V} \frac{m D^{1}\left[\tilde{u}-a_{12} \tilde{v} / a_{11}\right]+D^{2}[\tilde{v}]}{\tilde{H}[(\tilde{u}, \tilde{v})]} .
$$

Since the map generated by $P$ is invertible, it follows that the family of possible $(k-1)$-tuples $V$ is identical to the family of $(k-1)$-tuples $P V$; thus, in view of (3.15), we have

$$
\begin{aligned}
\mu_{k}(m)= & \sup _{\substack{V \subseteq\left[H_{0}^{1}(\Omega)\right]^{2} \\
V=\left\{\mathbf{w}_{1}, \ldots, \mathbf{w}_{k-1}\right\}}} \inf _{(u, v) \perp_{H} V} \frac{m D^{1}[u]+D^{2}[v]}{H[(u, v)]} \\
= & \sup _{\substack{\tilde{V} \subset\left[H_{0}^{1}(\Omega)\right]^{2} \\
\tilde{V}=\left\{\mathbf{y}_{1}, \ldots, \mathbf{y}_{k-1}\right\}}} \inf _{(\tilde{u}, \tilde{v}) \perp_{\tilde{H}} \tilde{V}} \frac{m D^{1}\left[\tilde{u}-a_{12} \tilde{v} / a_{11}\right]+D^{2}[\tilde{v}]}{\tilde{H}[(\tilde{u}, \tilde{v})]} .
\end{aligned}
$$

We will now drop the tildes on the $(\tilde{u}, \tilde{v})$ terms in the second line of (5.6) and estimate the terms occurring there. We have

$$
\begin{aligned}
D^{1}\left[u-\frac{a_{12} v}{a_{11}}\right] & =\int \sum_{i, j=1}^{N} A_{i j}^{1}\left[u_{x_{i}}-\left(\frac{a_{12} v}{a_{11}}\right)_{x_{i}}\right]\left[u_{x_{j}}-\left(\frac{a_{12} v}{a_{11}}\right)_{x_{j}}\right] \\
& =D^{1}[u]+D^{1}\left[\left(\frac{a_{12} v}{a_{11}}\right)\right]-2 \int \sum_{i, j=1}^{N} A_{i j}^{1} u_{x_{i}}\left(\frac{a_{12} v}{a_{11}}\right)_{x_{j}} .
\end{aligned}
$$


By condition (i), the map $(\boldsymbol{\xi}, \boldsymbol{\eta}) \mapsto \sum_{i, j=1}^{N} A_{i j}^{1}(x) \xi_{i} \eta_{j}$ is an inner product on $\mathbf{R}^{N}$ for any fixed $x$, so

$$
\begin{aligned}
& \left|2 \sum_{i, j=1}^{N} A_{i j}^{1} u_{x_{i}}\left(\frac{a_{12} v}{a_{11}}\right)_{x_{j}}\right| \\
& \quad \leq m^{1 / 2} \sum_{i, j=1}^{N} A_{i j}^{1} u_{x_{i}} u_{x_{j}}+m^{-1 / 2} \sum_{i, j=1}^{N} A_{i j}^{1}\left(\frac{a_{12} v}{a_{11}}\right)_{x_{i}}\left(\frac{a_{12} v}{a_{11}}\right)_{x_{j}}
\end{aligned}
$$

and hence by (5.7) and (5.8) we have

$$
\begin{aligned}
& \left(1-m^{1 / 2}\right) D^{1}[u]+\left(1-m^{-1 / 2}\right) D^{1}\left[\left(a_{12} v / a_{11}\right)\right] \leq D^{1}\left[u-\left(a_{12} v / a_{11}\right)\right], \\
& D^{1}\left[u-\left(a_{12} v / a_{11}\right)\right] \leq\left(1+m^{1 / 2}\right) D^{1}[u]+\left(1+m^{-1 / 2}\right) D^{1}\left[\left(a_{12} v / a_{11}\right)\right] .
\end{aligned}
$$

By assumption $a_{12} / a_{11} \in C^{1}(\bar{\Omega})$ so there are constants $K_{1}$ and $K_{2}$ such that

$$
0 \leq\left(a_{12} / a_{11}\right)^{2} \leq K_{1} \quad \text { and } \quad 0 \leq \sum_{i, j=1}^{N} A_{i j}^{1}\left(\frac{a_{12}}{a_{11}}\right)_{x_{i}}\left(\frac{a_{12}}{a_{11}}\right)_{x_{j}} \leq K_{2} \text {. }
$$

Also, by $(4.2), 0 \leq D^{1}[v] \leq\left(E_{1} / e_{2}\right) D^{2}[v]$. Finally,

$$
\gamma_{1}(-\Delta, 1) \int v^{2} \leq \int|\nabla v|^{2} \leq\left(1 / e_{2}\right) D^{2}[v]
$$

for any $v \in H_{0}^{1}(\Omega)$ by Poincaré's inequality. Thus, using (5.10), (5.11) and (4.2),

$$
\begin{aligned}
& 0 \leq D^{1}\left[\left(\frac{a_{12} v}{a_{11}}\right)\right]=\int \sum_{i, j=1}^{N} A_{i j}^{1}\left[\left(\frac{a_{12}}{a_{11}}\right)_{x_{i}} v+\left(\frac{a_{12}}{a_{11}}\right) v_{x_{i}}\right] \\
& \times\left[\left(\frac{a_{12}}{a_{11}}\right)_{x_{j}} v+\left(\frac{a_{12}}{a_{11}}\right) v_{x_{j}}\right] \\
& =\int \sum_{i, j=1}^{N} A_{i j}^{1}\left(\frac{a_{12}}{a_{11}}\right)_{x_{i}}\left(\frac{a_{12}}{a_{11}}\right)_{x_{j}} v^{2} \\
& +2 \int \sum_{i, j=1}^{N} A_{i j}^{1}\left[\left(\frac{a_{12}}{a_{11}}\right)_{x_{i}} v\right]\left[\left(\frac{a_{12}}{a_{11}}\right) v_{x_{j}}\right] \\
& +\int \sum_{i, j=1}^{N} A_{i j}^{1}\left(\frac{a_{12}}{a_{11}}\right)^{2} v_{x_{i}} v_{x_{j}} \\
& \leq 2 \int \sum_{i, j=1}^{N} A_{i j}^{1}\left(\frac{a_{12}}{a_{11}}\right)_{x_{i}}\left(\frac{a_{12}}{a_{11}}\right)_{x_{j}} v^{2} \\
& +2 \int \sum_{i, j=1}^{N} A_{i j}^{1}\left(\frac{a_{12}}{a_{11}}\right)^{2} v_{x_{i}} v_{x_{j}} \\
& \leq 2 K_{2} \int v^{2}+2 K_{1} D^{1}[v] \\
& \leq\left(2\left[K_{2}\left(\gamma_{1}(-\Delta, 1)\right)^{-1}+K_{1} E_{1}\right] / e_{2}\right) D^{2}[v] \text {. }
\end{aligned}
$$


For $m<1$ and $K_{3}$ sufficiently large (but independent of $m$ ) we have by (5.9) and $(5.12)$

$$
D^{1}\left[u-\left(a_{12} v / a_{11}\right)\right] \geq\left(1-m^{1 / 2}\right) D^{1}[u]+\left(1-m^{-1 / 2}\right) K_{3} D^{2}[v]
$$

and

$$
D^{1}\left[u-\left(a_{12} v / a_{11}\right)\right] \leq\left(1+m^{1 / 2}\right) D^{1}[u]+\left(1+m^{-1 / 2}\right) K_{3} D^{2}[v]
$$

Let

$$
\underline{\mu}_{k}(m)=\sup _{\substack{V \subseteq\left[H_{0}^{1}(\Omega)\right]^{2} \\ V=\left\{\mathbf{w}_{1}, \ldots, \mathbf{w}_{k-1}\right\}}} \inf _{(u, v) \perp_{\tilde{H}} V} \frac{m\left(1-m^{1 / 2}\right) D^{1}[u]+\left(1+m\left(1-m^{-1 / 2}\right) K_{3}\right) D^{2}[v]}{\tilde{H}[(u, v)]}
$$

and let

$$
\bar{\mu}_{k}(m)=\sup _{\substack{V \subseteq\left[H_{0}^{1}(\Omega)\right]^{2} \\ V=\left\{\mathbf{w}_{1}, \ldots, \mathbf{w}_{k-1}\right\}}} \inf _{(u, v) \perp_{\tilde{H}} V} \frac{m\left(1+m^{1 / 2}\right) D^{1}[u]+\left(1+m\left(1+m^{-1 / 2}\right) K_{3}\right) D^{2}[v]}{\tilde{H}[(u, v)]}
$$

where $m$ is chosen sufficiently small so that $1+m\left(1-m^{-1 / 2}\right) K_{3}>0$. Then by (5.6), (5.13) and (5.14) we have

$$
\underline{\mu}_{k}(m) \leq \mu_{k}(m) \leq \bar{\mu}_{k}(m) .
$$

From (5.15) and (5.16) we have that $\underline{\mu}_{k}(m)$ and $\bar{\mu}_{k}(m)$ are the $k$ th eigenvalues for

$$
m\left(1-m^{1 / 2}\right) L_{1} u=\underline{\mu} a_{11} u, \quad\left(1+m\left(1-m^{-1 / 2}\right) K_{3}\right) L_{2} v=\underline{\mu} a_{\infty} v
$$

and

$$
m\left(1+m^{1 / 2}\right) L_{1} u=\bar{\mu} a_{11} u, \quad\left(1+m\left(1+m^{-1 / 2}\right) K_{3}\right) L_{2} v=\bar{\mu} a_{\infty} v
$$

respectively. Hence, the sequence $\left\langle\underline{\mu}_{k}(m)\right\rangle$ is precisely the set

$$
\left\{m\left(1-m^{1 / 2}\right) \gamma_{i}\left(L_{1}, a_{11}\right),\left[1-m^{1 / 2} K_{3}+m K_{3}\right] \gamma_{j}\left(L_{2}, a_{\infty}\right)\right\}
$$

written in increasing order; similarly, $\left\langle\bar{\mu}_{k}(m)\right\rangle$ is the set $\left\{m\left(1+m^{1 / 2}\right) \gamma_{i}\left(L_{1}, a_{11}\right)\right.$, $\left.\left[1+m^{1 / 2} K_{3}+m K_{3}\right] \gamma_{j}\left(L_{2}, a_{\infty}\right)\right\}$ written in increasing order, since the systems (5.18) and (5.19) are uncoupled. We wish to show that for all $j \in Z^{+}$and all $m$ sufficiently small there is a $k \in Z^{+}$such that

$$
\left[1-m^{1 / 2} K_{3}+m K_{3}\right] \gamma_{j}\left(L_{2}, a_{\infty}\right) \leq \underline{\mu}_{k}(m) \leq \mu_{k}(m)
$$

and

$$
\mu_{k}(m) \leq \bar{\mu}_{k}(m) \leq\left[1+m^{1 / 2} K_{3}+m K_{3}\right] \gamma_{j}\left(L_{2}, a_{\infty}\right) .
$$

Such will be the case provided that whenever

$$
\underline{\mu}_{k}(m)=\left[1-m^{1 / 2} K_{3}+m K_{3}\right] \gamma_{j}\left(\lambda_{2}, a_{\infty}\right)
$$

then for some $r \geq 0, \bar{\mu}_{k+r}(m)=\left[1+m^{1 / 2} K_{3}+m K_{3}\right] \gamma_{j}\left(L_{2}, a_{\infty}\right)$. Thus, we want to have the number of eigenvalues of (5.19) less than $\left[1+m^{1 / 2} K_{3}+m K_{3}\right] \gamma_{j}\left(L_{2}, a_{\infty}\right)$ to be at least as large as the number of eigenvalues of (5.18) less than

$$
\left[1-m^{1 / 2} K_{3}+m K_{3}\right] \gamma_{j}\left(L_{2}, a_{\infty}\right) \text {. }
$$


The number of such eigenvalues arising from the second equation in either system will be $j-1$, so we need only show that if

$$
m\left(1-m^{1 / 2}\right) \gamma_{i}\left(L_{1}, a_{11}\right) \leq\left[1-m^{1 / 2} K_{3}+m K_{3}\right] \gamma_{j}\left(L_{2}, a_{\infty}\right)
$$

then

$$
m\left(1+m^{1 / 2}\right) \gamma_{i}\left(L_{1}, a_{11}\right) \leq\left[1+m^{1 / 2} K_{3}+m K_{3}\right] \gamma_{j}\left(L_{2}, a_{\infty}\right)
$$

Now, (5.22) implies that

$$
m\left(1+m^{1 / 2}\right) \gamma_{i}\left(L_{1}, a_{11}\right) \leq\left[\frac{1+m^{1 / 2}}{1-m^{1 / 2}}\right]\left[1-m^{1 / 2} K_{3}+m K_{3}\right] \gamma_{j}\left(L_{2}, a_{\infty}\right)
$$

so (5.23) follows provided that we have

$$
\left[\frac{1+m^{1 / 2}}{1-m^{1 / 2}}\right]\left[1-m^{1 / 2} K_{3}+m K_{3}\right] \leq\left[1+m^{1 / 2} K_{3}+m K_{3}\right]
$$

Since we assume $m<1,(5.24)$ is equivalent to the corresponding inequality multiplied on both sides by $\left(1-m^{1 / 2}\right)$. Performing the multiplication and simplifying yields the equivalent inequality

$$
m K_{3} \leq K_{3}-1 \text {. }
$$

We may choose $K_{3}>1$; then (5.25) holds and hence (5.22) implies (5.23), provided $m$ is sufficiently small. Thus, for each $j \in Z^{+}$and for all $m$ sufficiently small, there is a point $(\lambda, \mu)=(\mu / m, \mu) \in \Sigma$ such that

$$
\left[1-m^{1 / 2} K_{3}+m K_{3}\right] \gamma_{j}\left(L_{2}, a_{\infty}\right) \leq \mu \leq\left[1+m^{1 / 2} K_{3}+m K_{3}\right] \gamma_{j}\left(L_{2}, a_{\infty}\right)
$$

Since we may choose $\mu$ to depend continuously on $m$, we may take $\lambda$ to be a continuous function of $m$ with $\lambda \rightarrow \infty$ as $m \rightarrow 0$. The intermediate value theorem then implies that $\lambda$ takes on all sufficiently large values. For $m$ sufficiently small, (5.26) gives $\left|\gamma_{j}\left(L_{2}, a_{\infty}\right)-\mu\right|<\delta$; so the points $(\lambda, \mu)$ form the desired set $S_{j}$.

\section{REFERENCES}

1. J. C. Alexander and S. S. Antman, Global and local behavior of bifurcating multidimensional continua of solutions for multiparameter nonlinear eigenvalue problems, Arch. Rational Mech. Anal. 76 (1981), 339-354.

2. __ Global behavior of solutions of nonlinear equations depending on infinite dimensional parameters, Indiana Univ. Math. J. 32 (1983), 39-62.

3. K. J. Brown and J. C. Eilbeck, Bifurcation, stability diagrams, and varying diffusion coefficients in reaction-diffusion equations, Bull. Math. Biol. 44 (1982), 87-102.

4. R. S. Cantrell, A homogeneity condition guaranteeing bifurcation in multiparameter nonlinear eigenvalue problems, Nonlinear Anal. 8 (1984), 159-169.

5. __, On coupled multiparameter nonlinear elliptic systems, Trans. Amer. Math. Soc. 294 (1986), 263-285.

6. R. S. Cantrell and C. Cosner, On the steady-state problem for the Volterra-Lotka competition model with diffusion, Houston J. Math. (to appear).

7. __ On the uniqueness of positive solutions in the Lotka-Volterra competition model with diffusion, Houston J. Math. (to appear).

8. R. S. Cantrell and K. Schmitt, On the eigenvalue problem for coupled elliptic systems, SIAM J. Math. Anal. 17 (1986), 850-862.

9. R. Courant and D. Hilbert, Methods of mathematical physics, Vol. I, Interscience, New York, 1965. 
10. P. M. Fitzpatrick, I. Massabo and J. Pejsachowicz, Global several-parameter bifurcation and continuation theorems: a unified approach via complementing maps, Math. Ann. 263 (1983), 61-73.

11. - On the covering dimension of the set of solutions of some nonlinear equations, Trans. Amer. Math. Soc. 296 (1986), 777-798.

12. D. Henry, Topics in nonlinear analysis, Trabalho de Matematica No. 192, Universidade de Brasilia, 1982.

13. J. Ize, I. Massabo, J. Pejsachowicz and A. Vignoli, Structure and dimension of global branches of solutions to multiparameter nonlinear equations, Trans. Amer. Math. Soc. 291 (1985), 383-435.

14. M. H. Protter, The generalized spectrum of second order elliptic systems, Rocky Mountain J. Math. 9 (1979), 503-518.

15. F. Rellich, Perturbation theory of eigenvalue problems, Gordon and Breach, New York, 1969.

16. J. T. Schwartz, Nonlinear functional analysis, Gordon and Breach, New York, 1969.

17. K. Uhlenbeck, Generic properties of eigenfunctions, Amer. J. Math. 98 (1976), 1059-1078.

Department of Mathematics and Computer Science, University of Miami, CORAL GABLES, FLORIDA 33124 